\title{
Encephalopathy with intracerebral calcification, white matter lesions, growth hormone deficiency, microcephaly, and retinal degeneration: two sibs confirming a probably distinct entity
}

\author{
Carsten G Bönnemann, Peter Meinecke, Horst Reich
}

\begin{abstract}
Two sibs with an encephalopathy, including intracerebral calcification and white matter lesions, dwarfism owing to growth hormone deficiency, and retinal degeneration are reported. The onset of the disease in both patients occurred with retardation of motor development during the first year of life. Later, dwarfism, mental retardation, spasticity, ataxia, and retinal degeneration became apparent. These cases probably represent some form of connatal leucodystrophy. The differential diagnosis is discussed.
\end{abstract}

Encephalopathies with intracranial calcification in infants and children represent a heterogeneous group of diseases of different pathogenesis and aetiology. ${ }^{1}$ Recently, Billard et al ${ }^{2}$ proposed a classification of cases of calcification of the basal ganglia of unknown cause in children, which was formerly called Fahr syndrome. These authors delineated at least four different entities, of which three are presumed to be of genetic aetiology.

We report two sibs with such an encephalopathy and discuss these cases in view of the new classification.

Altonaer Kinderkrankenhaus, Bleickenallee 38, D2000 Hamburg 50, Germany.

C G Bönnemann

Abteilung Medizinische Genetik, Altonaer Kinderkrankenhaus, Bleickenallee 38, D-2000 Hamburg 50, Germany.

P Meinecke

St Marienhospital, Kinderabteilung, Marienstrasse 6, D-2848 Vechta, Germany.

$\mathrm{H}$ Reich

Correspondence to Dr Meinecke.

Received for publication 1 October 1990.

Revised version accepted for publication 13 February 1991.

\section{Case reports}

FAMILY HISTORY

The parents were unrelated but originated from the same small town in northern Germany. After one female stillbirth in the seventh month of gestation and one abortion, case 1 was born. After a second abortion case 2 was born, whose twin brother was healthy. The family history is otherwise unremarkable.

\section{CASE 1}

This female infant was born at term after an uneventful pregnancy, with a birth weight of $2650 \mathrm{~g}$ ( $-2 \mathrm{SD})$ and length $47 \mathrm{~cm}(-2 \mathrm{SD})$. Development during the first year of life was retarded; she did not sit until 12 months, spoke her first words at 18 months, and started to walk at 26 months of age. Her walking was described as being severely ataxic, but deep tendon reflexes were normal. Later she developed signs of spasticity in the legs. Her language skills remained poor, so she attended a special education programme at 8 years of age.

At the age of 16 months short stature became apparent $(71 \mathrm{~cm},-3 \mathrm{SD})$, which by the end of the third year had become even more pronounced $(84 \mathrm{~cm},-3.5 \mathrm{SD})$. At that time growth hormone (GH) deficiency was diagnosed with abnormal arginine and insulin provocation tests. Hypothyroidism was also found. Substitution with GH and thyroxine was started. Skeletal age was markedly retarded, being equivalent to 1.5 years at 4 years of age. Her height at the age of 10 years was $118 \mathrm{~cm}$ $(-4 \mathrm{SD})$ and weight was $20.5 \mathrm{~kg}$ (50th centile according to height). Her head circumference was not recorded. $\mathrm{G}$ banded chromosomes were normal.

The condition of the ocular fundi was not reported, but she required glasses for hyperopia. EEG showed abnormal activity intermittently with an apparent focus over the left temporoparietal region.

The girl died at 10 years from a fulminant illness starting with vomiting and loss of consciousness. 
Necropsy showed evidence of massive pneumonia and hypoplasia of the pituitary gland, adrenals, and uterus. The brain showed massive oedema (weight $1260 \mathrm{~g}$ ). Multiple calcifications, partly perivascular in distribution, could be seen in the pituitary gland, hypothalamus, and basal ganglia. Evidence of demyelination was found in the occipital areas. The presumed cause of death was pneumonia with consecutive brain oedema because of adrenal insufficiency.

\section{CASE 2}

This girl was born at term by caesarean section after an uneventful pregnancy. Her birth weight was $2880 \mathrm{~g}(-2 \mathrm{SD})$. Early development was retarded; she did not sit until 11 months of age and she never learned to walk, although she was able to crawl. Language skills were confined to a few words.

Severe growth retardation became apparent early on, as her height was only $76 \mathrm{~cm}(-4.5 \mathrm{SD})$ at 28 months and $91 \mathrm{~cm}(-4.7 \mathrm{SD})$ at 5 years. Bone age at that time was equivalent to a 2.5 year old. Her head circumference was $46.5 \mathrm{~cm}(-3 \mathrm{SD})$. No dysmorphic features could be seen. At that time (5 years) GH deficiency was diagnosed using the arginine and insulin provocation tests. Thyroid hormones and cortisol were normal as was parathyroid hormone (PTH), serum calcium, and phosphorus. Serum amino acid chromatography and TORCH titres were negative, and routine examination of the spinal fluid was normal. GH substitution was started.

The EEG was abnormal with diffuse hypersynchronous activity. A radiograph of the skull showed bilateral symmetrical calcifications and a CT scan of the brain at 26 months showed these calcifications in the periventricular and subcortical white matter and also in the pons. The basal ganglia were virtually free of calcification initially (fig 1). However, a repeat scan nearly three years later clearly showed heavy calcification in the putamen (fig 2) and calcification of the deep cerebellar nuclei could now also be seen. MRI studies at the age of 6.5 years showed increased signal on $\mathrm{T} 2$ weighted images in the periventricular white matter, as well as atrophy of the cerebellar vermis, cerebellar hemispheres, and brainstem (fig 3).

Her development continued to be severely retarded and she showed increasing spasticity in the legs, with hypotonia in the arms and trunk. Some degree of ataxia was also noted. Deep tendon reflexes were hyperactive in the legs.

At the age of 6.5 years her height was $98 \mathrm{~cm}$ ( $-4.4 \mathrm{SD})$, her weight $16 \mathrm{~kg}$, and her head circumference $47 \mathrm{~cm}$ ( $-3 \mathrm{SD}$ ). Funduscopy showed heavy pigmentation. A diagnosis of tapetoretinal degeneration was made. At the age of 8.5 years partial atrophy of the optic nerve was noted.

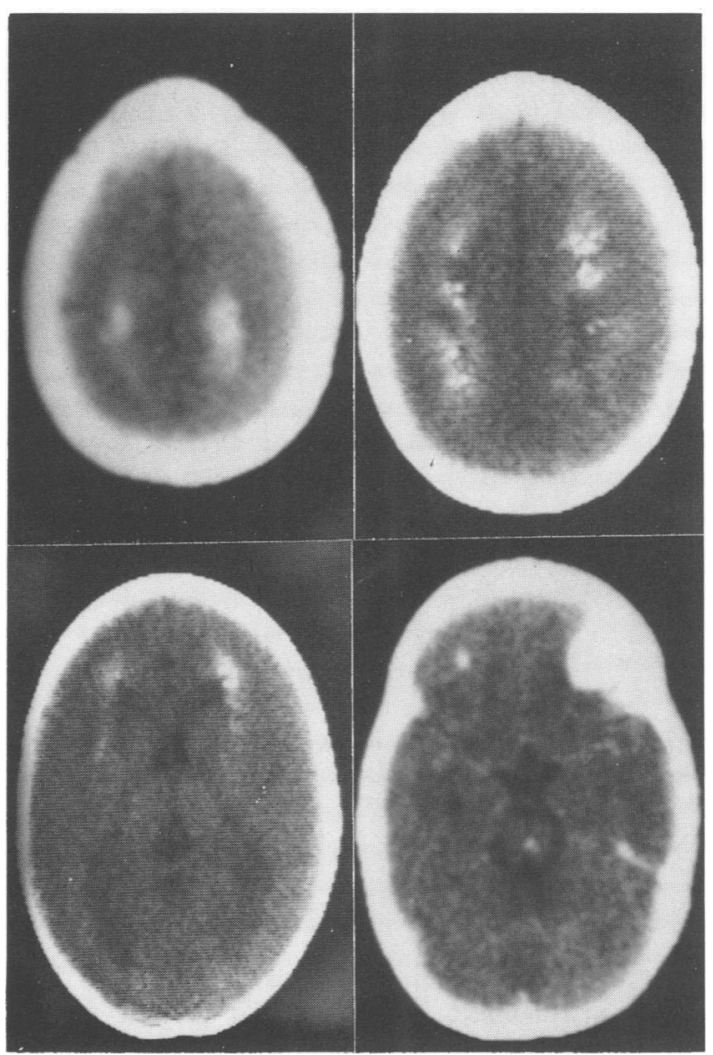

Figure 1 Case 2. CT scan of the brain at 26 months showing extensive calcification of the periventricular and subcortical white matter. Calcification is also seen in the pons, whereas the basal ganglia are virtually free of calcification. (Postcontrast scan.)

At the age of 9 years she died during an episode of pneumonia with status epilepticus progressing to brain death. Necropsy was not performed.

\section{Discussion}

Recently, Billard $e t a l^{2}$ proposed a classification of encephalopathies of childhood with calcification of the basal ganglia as a distinguishing feature. As they did not consider diseases with intracerebral calcification in general, several of the known calcifying diseases, ${ }^{1}$ such as neoplasms and neurocutaneous diseases, and the more common prenatal infections, such as toxoplasmosis and cytomegalic inclusion disease, were not included. Also not considered were a number of diseases of known, or presumed known, pathogenesis producing calcification of the basal ganglia. These are, among others, disorders of calcium metabolism, cardiovascular events, methotrexate encephalopathy, biotinidase deficiency, carbonic 


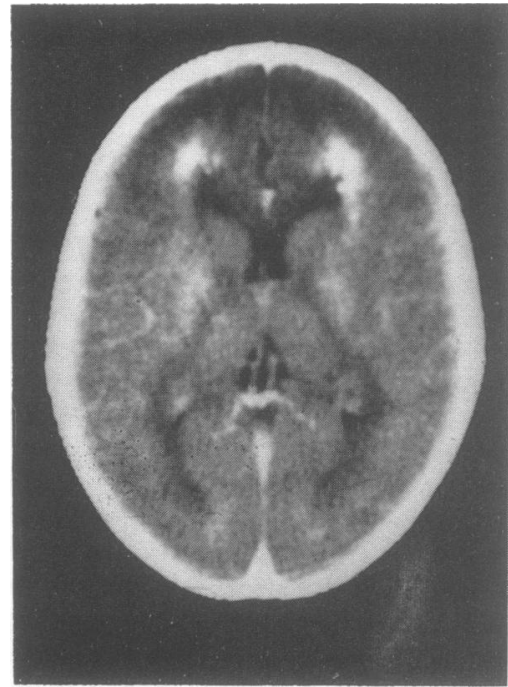

Figure 2 Case 2. Second CT scan taken three years after the first scan (fig 1). In addition to the calcification already described, heavy calcification is found in the putamen as well. (Postcontrast scan.)

anhydrase II deficiency, Down's syndrome, Cockayne's syndrome, possibly Krabbe's disease, neurofibromatosis, mitochondrial encephalomyopathies, and AIDS. Instead, the classification by Billard et $a l^{2}$ is specifically concerned with cases of encephalopathy with calcification of the basal ganglia of unknown pathogenesis, which thus present considerable difficulties for both nosological categorisation and for genetic counselling of the sporadic case. These cases, formerly collectively called Fahr disease, are now divided into four groups by these authors. However, some of the authors' classification of older published cases may be seen as controversial. ${ }^{3}$

In short, group 1 represents a slowly progressive encephalopathy of presumably autosomal recessive inheritance associated with microcephaly, dwarfism, retinal degeneration or optic atrophy, and symmetrical patchy demyelination. Group 2 comprises patients with a sporadic, stationary encephalopathy resulting from an unknown prenatal insult. Group 3 is characterised by a progressive encephalopathy with chronic spinal fluid lymphocytosis, originally described by Aicardi and Goutières. ${ }^{+}$The fourth group is of autosomal dominant inheritance, with mild neurological symptoms in the oldest family members only. ${ }^{5}$ Two cases observed by Billard et $a l^{2}$ remained unclassified and some additional published cases are also not easily fitted into this classification. ${ }^{367}$

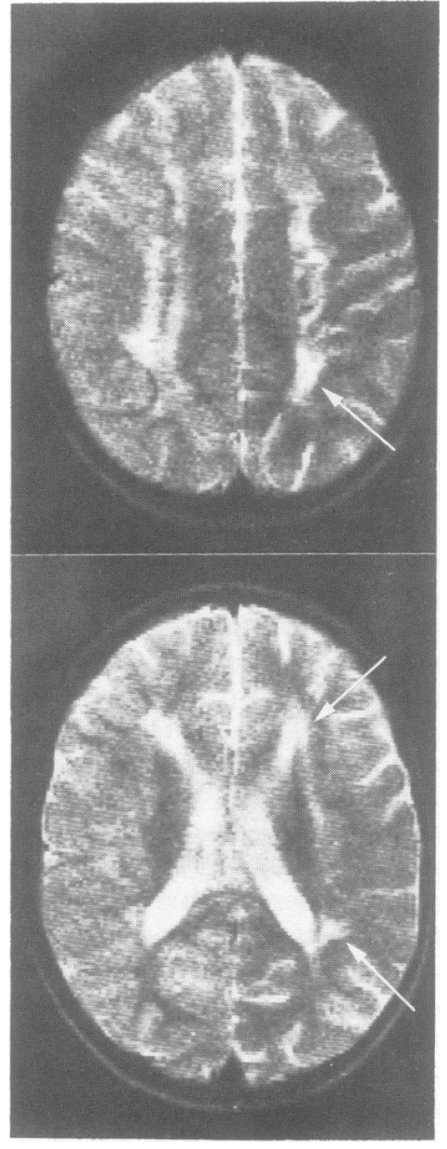

Figure 3 Case 2. MRI scan at 6.5 years. High signal on $T 2$ weighted images is seen in the periventricular white matter (arrows).

Our cases showed microcephaly (documented in case 2 only), tapetoretinal degeneration (documented in case 2 only), severe growth hormone deficiency with dwarfism in both, hypothyroidism in case 1 , calcification of the basal ganglia in case 1 , and periventricular calcification only in case 2 . Onset was during the first year of life and progression was slow. On neurological examination they showed mental retardation, slurred speech, a spastic paraparesis, and ataxia. Pathological and MRI studies suggested dysmyelination. Autosomal recessive inheritance is strongly suggested by the familial occurrence. Although the clinical data are unfortunately not complete, we think these sibs most resemble the first group described by Billard $e t a l{ }^{2}$ and the cases of Melchior et al. ${ }^{8}$ An important differential diagnosis for patients like ours is Cockayne's syndrome, a DNA repair defect syndrome with dwarfism, retinitis pigmentosa, and mental retardation. However, 
the age of onset in Cockayne's syndrome is around the age of 2 to 3 years, which is clearly later than in the cases under discussion. More importantly they showed a characteristic facial phenotype, which can be readily recognised by the experienced examiner. This also holds true for an early onset variant of Cockayne's syndrome in which cataracts, contractures, and kyphosis are additional features. ${ }^{910} \mathrm{~A}$ history of light sensitivity can usually be elicited. Thus, our cases can clearly be distinguished from Cockayne's syndrome.

Calcification of the basal ganglia in addition to retinitis pigmentosa can also be found in the mitochondrial encephalomyopathies, especially in the Kearns-Sayre syndrome. ${ }^{112}$ Again, age of onset tends to be later, dwarfism is not a feature of this condition, and mental retardation is not prominent. However, ataxia might well be found, but external ophthalmoplegia and heart block, characteristic features of this disease, were not found in our patients. The combination of tapetoretinal degeneration with calcification of the basal ganglia, together with retarded growth, can be found in an additional report. ${ }^{13}$ The symptoms in these cases seem to be the result of some defect in PTH function, the resulting bone disease being responsible for the short stature observed.

In conclusion, our cases conform to group 1 of the classification of Billard et al. ${ }^{1}$ They show some interesting features; as seen in our first case, the endocrinological abnormalities are not necessarily confined to growth hormone only. To explain the additional hypothyroidism, adrenal hypoplasia, and hypoplasia of the uterus, a more generalised hypothalamic/pituitary dysfunction is possible, although detailed endocrinological data for this patient are unfortunately not available. Detailed endocrinological studies would be desirable for every patient with this condition. Also of note is the different extent of the calcification in the two patients, one (case 2) showing evolution of calcification of the basal ganglia over the course of a few years. This should be kept in mind if a sporadic patient with this disease is seen; calcification of the basal ganglia may not be necessary for the diagnosis of the disease.

At present the pathogenesis of this probably autosomal recessive condition can only be a matter of speculation. In view of the white matter changes seen on neuropathological examination, as well as on MRI scanning, some form of connatal leucodystrophy seems to be likely. This remains to be shown in future studies.

We wish to thank Professor Dr G Schellong, Münster, Germany, for providing clinical data.

1 Kendall B, Cavanagh N. Intracranial calcification in paediatric computed tomography. Neuroradiology 1986;28:324-30.

2 Billard C, Dulac O, Bouloche J, et al. Encephalopathy with calcifications of the basal ganglia in children. A reappraisal of Fahr's syndrome with respect to 14 new cases. Neuropediatrics 1989;20:12-19.

3 Baraitser M, Brett EM, Piesowicz AT. Microcephaly and intracranial calcification in two brothers. $\mathcal{f}$ Med Genet 1983;20:210-12.

4 Aicardi J, Goutières F. A progressive familial encephalopathy in infancy with calcifications of the basal ganglia and chronic cerebrospinal fluid lymphocytosis. Ann Neurol 1984;15:4954.

5 Boller F, Boller M, Gilbert J. Familial idiopathic cerebral calcifications. I Neurol Neurosurg Psychiatry 1977;40:280-5.

6 Burn J, Wickramasinghe HT, Harding B, Baraitser M. A syndrome with intracranial calcification and microcephaly in two sibs, resembling intrauterine infection. Clin Genet 1986;30:112-16.

7 Ishitsu T, Chikazawa S, Matsuda I. Two siblings with microcephaly associated with calcification of cerebral white matter. fpn f Hum Genet 1985;30:213-17.

8 Melchior JC, Benda CE, Yakovlev PI. Familial idiopathic cerebral calcifications in childhood. Am $尹$ Dis Child 1960;99:787-803.

9 Patton MA, Gianelli F, Francis AJ, Baraitser M, Harding B, Williams AJ. Early onset Cockayne's syndrome: case reports with neuropathological and fibroblast studies. $f$ Med Genet 1989;26:154-9.

10 Talwar D, Smith SA. CAMFAK syndrome: a demyelinating inherited disease similar to Cockayne syndrome. $\mathrm{Am} \mathcal{F} \mathrm{Med}$ Genet 1989;34:194-8.

11 Dewhurst AG, Hall D, Schwartz MS, McKeran RO. KearnsSayre syndrome, hypoparathyroidism, and basal ganglia calcification. F Neurol Neurosurg Psychiatry 1986;49:1323-4.

12 Robertson WC, Viseskul C, Lee YE, Lloyd RV. Basal ganglia calcification in Kearns-Sayre syndrome. Arch Neurol 1979;36:711-13.

13 Hammerstein W, Bischof G, Keck E. A tapetoretinal degeneration with symmetrical calcifications of the basal ganglia. Eur Neurol 1982;21:249-55. 